N. FABIAN KLEIMEIER, MATTHEW JAMES ABPLANALP, Department of Chemistry, University of Hawaii at Manoa, Honolulu, HI, USA; REBECCAA N. JOHNSON, SAMER GOZEM, Department of Chemistry, Georgia State University, Atlanta, GA, USA; RALF INGO KAISER, Department of Chemistry, University of Hawaii at Manoa, Honolulu, HI, USA.

Over 200 molecules have been detected in the interstellar medium (ISM) with close to one third considered to be complex organic molecules (COMs), molecules containing six or more atoms. Gas-phase reaction networks of ion-molecule hese models fail to explain the synthesis of ubiquitous COMs with predicted abundances several orders of magnitude lower compared to observations in the ISM, such as in Sagittarius B2. Over the last decades astrophysical laboratory simulation experiments have shown that some of these COMs are formed via interaction of ionizing radiation within simple ices deposited desorption was utilized to sublime the traviolet ionization coupled with a reflectron time-of-flight mass spectrometer (PI-ReTOF-MS). The use of PI-ReTOF-MS allows for the selective ionization and identification of structural isomers of COMs. Here, we report that the key COM propynal ( $\mathrm{HCCCHO}$ ) and cyclopropenone $\left(\mathrm{c}-\mathrm{C}_{3} \mathrm{H}_{2} \mathrm{O}\right)$, which have both been detected in the ISM, can be synthesized within interstellar ices containing carbon monoxide $(\mathrm{CO})$ and acetylene $\left(\mathrm{C}_{2} \mathrm{H}_{2}\right)$ at temperatures as low as $5 \mathrm{~K}$. This is accomplished via non-equilibrites. ing interstellar ices. Furthermore, cyclic COMs may act as tracers for non-equilibrium chemical processes at $10 \mathrm{~K}$ involving experiments, such as yield, branching ratio, and chemical and temperature conditions, into astrochemical models accounting for non-equilibrium has been shown to greatly improve predicted abundances.

\title{
THE MILLIMETERWAVE SPECTRUM OF SUCCINONITRILE
}

CARLOS CABEZAS, CELINA BERMÚDEZ, Instituto de Fisica Fundamental, CSIC, Madrid, Spain; JUAN DANIEL GALLEGO, Centro Astronómico de Yebes, Observatorio Astronómico Nacional, Yebes, Guadalajara, la Materia, (IEM-CSIC), Madrid, Spain; BELÉN TERCERO, OAN-IGN, Observatorio Astronómico Nacional Madrid, Spain; ROSA LEBRON, JESUUS EDUARDO QUINTANILLA, Instituto Quimica-Física Rocasolano, CSIC, Madrid, Spain; ANA CRISTINA SORIA, Análisis Instrumental y Quimica Ambiental, CSIC, Madrid Spain; JOSE CERNICHARO, Instituto de Fisica Fundamental, CSIC, Madrid, Spain.

Only two dinitriles, the protonated cyanogen $\mathrm{NCCNH}^{+}$and one isocyanonitrile, $\mathrm{NCNC}$ have been detected in the intersellar medium (ISM) among about 200 other compounds, despite nitriles constitute almost $20 \%$ of the molecules observed on their rotational spectra since most of them do not present permanent dipole moment. Succinonitrile, $\mathrm{NCCH}_{2} \mathrm{CH}_{2} \mathrm{CN}$ is one of the simplest dinitriles and it could be a candidate molecule to be observed in the ISM. The rotational spectrum for succinonitrile has been previously observed in the $2-78 \mathrm{GHz}$ frequency region by Jahn et al., ${ }^{a}$ but these measurements were Imited to the ground state. In this talk we report the study of succinonitrile and its low lying excited vibrational states using a
newly built broadband Fourier transform millimeter wave spectrometer ${ }^{b}$ equipped with Q-band (31.5-50 GHz) and W-band 72-116 GHz) receivers, similar to those used in the radio-telescopes. The spectrometer is well suited for high resolution emission spectroscopy of molecules of astrophysical importance.

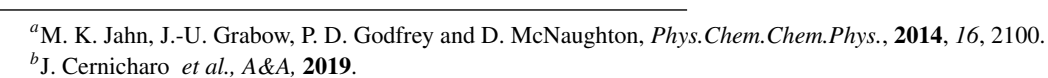

PHOTOPHYSICS AND ELECTRONIC STRUCTURE STUDIES OF PROTONATION OF QUINOLINE

$11: 30-11: 4$

HIRDYESH MISHRA, Department of Physics, Banaras Hindu University, Varanasi, Uttar Pradesh, India.

Study of the photophysics and electronic structure properties of quinoline and its derivatives have been the subject of considerable interest because of their commercial and pharmaceutical applications. Some of the quinoline derivatives have been found as a potential probes for measuring the polarity of microenvironment in chemical and biological systems. Diffuse interstellar bands in the emission spectrum of the interstellar medium, indicate the presence of quinoline and other small polycyclic aromatic nitrogen heterocycles, which readily dissociate under exposure to interstellar radiation. Since the quinostructure of quinoline in presence some external perturbation. Being isoelectronic with naphthalene, these molecules provide useful comparisons for checking the electronic and vibrational state assignments, ionization potentials, and other properties of the parent hydrocarbon. In addition, these molecules possess nonbonding electrons which give rise to n- $P i^{*}$ states. The location and characterization of these states are of both theoretical and practical significance. Further, solvents have an importan influence on the fluorescence property of N-heterocyclic compounds. Experimentally, Quinoline shows vibronic absorption spectrum and corresponding large Stoke shifted broad fluorescence emission spectrum having very low quantum yield an fluorescence decay become mono-exponential. To understand the vibronic structure of electronic absorption spectra and pho tophysics of protonation of quinoline, both vibronic and electronic structure studies of quinoline (Q) and protonated quinoline $(\mathrm{QH}+)$ were carried out along with vibrational calculations for absorption and fluorescence bands at B3LYP 6-311++G(d, p) level in ground and excited state by density functional methods (DFT) and (time-dependent density functional) TD-DFT methods respectively with the help of Gaussian 09 software. Normal mode mixing is taken into account by the Duschinsky transformation. The imation. Weakly dipole-allowed and dipole-forbidden transitions are treated within the Franck-Condon-Herzberg-Telle experiment results are found to understand the photo-physics of protonation of quinoline. 